\section{Crisis epilépticas post suspensión de Zolpidem}

\section{Epileptic seizures after zolpidem withdrawal}

\section{Señor Editor:}

El zolpidem es un hipnótico no benzodiacepínico, con alta afinidad al receptor GABA-A, perteneciente a la familia de las imidazopiridinas. Actúa como inductor del sueño.

En general es bien tolerado, seguro, y tendría un menor riesgo de abuso y dependencia que las benzodiacepinas ${ }^{1}$. Sin embargo, se han dado a conocer casos de abuso y dependencia ${ }^{2}$, y algunos reportes de pacientes que han presentado crisis epilépticas en relación a la suspensión brusca del zolpidem, usado en dosis altas $(150 \text { a } 600 \mathrm{mg})^{3-6}$.

Se presenta el caso de una paciente de 26 años con diagnóstico de trastorno bipolar, tratado con lamotrigina $300 \mathrm{mg}$. hasta hace 1 año y zolpidem $10 \mathrm{mg}$ entre 5 a 10 comprimidos cada noche.

En dos oportunidades ha suspendido completamente la ingesta de zolpidem y al día siguiente ha presentado crisis convulsivas tónico-clónicas generalizadas, con confusión post ictal de varios minutos de duración.

La cantidad de zolpidem que la paciente usa cada noche es variable porque refiere que frecuentemente presenta confusión mental después de tomarlo lo que la lleva a repetir la dosis.

Entre los exámenes tiene: resonancia magnética de cerebro, hemograma, perfil bioquímico, electrolitos plasmáticos, niveles hormonales tiroídeos, normales. El EEG con privación de sueño y solo $10 \mathrm{mg}$ de zolpidem revela un ritmo de fondo normal y ocasionales grafoelementos epileptogénicos bilaterales aislados.

Actualmente la paciente está con estrictas medidas de higiene de sueño, restricción del tiempo en cama y reducción progresiva del zolpidem, sin haber presentado nuevas crisis.

Los receptores GABA-A tienen varias subunidades. La alfa-1 está involucrada en los mecanismos del sueño y sería el lugar específico de acción del zolpidem.

Las benzodiacepinas tienen una afinidad no selectiva por todas las subunidades.

Es posible que dosis altas de zolpidem ocupen todas las subunidades de los receptores GABA-A, de manera similar a las benzodiacepinas y, por lo tanto su suspensión brusca produzca no solo insomnio sino que también ansiedad y crisis epilépticas.

El caso de esta paciente es un llamado de atención al uso crónico de estos medicamentos hipnóticos que se prescriben abundantemente en nuestro medio.
En las actuales circunstancias de la pandemia por el COVID-19, que estamos viviendo, el confinamiento y el temor al contagio por el virus, están produciendo frecuentes dificultades en la conciliación y mantención del sueño. Además, muchas personas están siendo afectadas por la disminución de sus ingresos, pérdida del trabajo, adaptación al teletrabajo, el cuidado de sus hijos pequeños, y su educación on-line, etc.

La ansiedad que pueden producir todos estos aspectos de la vida actual aumentan los trastornos de sueño, específicamente el insomnio. Es así como muchos médicos estamos detectando un incremento en el uso y abuso de los ansiolíticos y de los hipnóticos. Esto puede hacer que complicaciones infrecuentes, como el caso de esta paciente, se presenten mucho más.

José Luis Castillo ${ }^{1,2}$

${ }^{1}$ Servicio de Neurología, Neurocirugía y Neurorradiología intervencional, Clínica Santa María. Santiago, Chile. ${ }^{2}$ Departamento de Ciencias Neurológicas Oriente, Facultad de Medicina, Universidad de Chile. Santiago, Chile.

\section{Referencias}

1. Holm KJ, Goa KL. Zolpidem: an update of its pharmacology, therapeutic efficacy and tolerability in the treatment of insomnia. Drugs 2000; 59 (4): 865-89.

2. Krueger TH, Kropp S, Huber TJ. High-dose zolpidem dependence in a patient with chronic facial pain. Ann Pharmacother 2005; 39 (4): 773-4.

3. Aragona M. Abuse, dependence, and epileptic seizures after zolpidem withdrawal: review and case report. Clin Neuropharmacol 2000; 23 (5): 281-3.

4. Barrero-Hernández FJ, Ruiz-Veguilla M, López-López MI, Casado-Torres A. Epileptic seizures as a sign of abstinence from chronic consumption of zolpidem. Rev Neurol 2002; 34 (3): 253-6.

5. Cubala WJ, Landowski J. Sizure following sudden zolpidem withdrawal. Prog Neuropsychopharmacol Biol Psychiatry 2007; 31 (2): 539-40.

6. Seyed Alireza Haji Seyed Javadi, Farid Hajiali, Marjan Nassiri-Asl. Zolpidem dependency and withdrawal seizure: a case report study. Iran Red Crescent Med J 2014; 16 (11): 1-3.

Correspondencia a:

Dr. José Luis Castillo

Las Tranqueras Oriente 2565, Dpto. 31, Vitacura. Santiago, Chile. jcastill@med.uchile.cl 